\title{
THE DOUBLE COSETS OF A FINITE GROUP ${ }^{1}$
}

\section{J. S. FRAME}

1. Introduction. It is the purpose of this paper to study some of the properties of the double cosets of a finite group and to prove two main theorems which generalize the results of two previous papers by the author, ${ }^{2}$ giving some relations between the double cosets and the irreducible components of the permutation group generated by a given subgroup. We let $H$ be an arbitrary but fixed subgroup of order $h$ of a finite group $G$ of order $g, g=n h$, and we let $G_{H}$ be the permutation group of degree $n$ induced by right multiplication of the cosets $H S_{i}, i=1,2, \cdots, n$, by elements of $G$. When written as a group of permutation matrices and completely reduced, the group $G_{H}$ will have $r^{\prime}$ distinct irreducible components $\Gamma_{i}$ of degree $n_{i}$ and multiplicity $\mu_{i}^{H}$, and we may write

$$
G_{H}=\sum_{i=1}^{r^{\prime}} \stackrel{H}{\mu_{i}} \Gamma_{i} .
$$

Multiplication of a right coset $H G_{k}$ on the left by a single element of $G$ does not in general produce a right coset, but if each coset $H G_{k}$ is multiplified on the left by all the elements of a right coset $H S_{t}$ and the products are added, a transformation is obtained which carries each of the $n$ right cosets $H G_{k}$ into a collection of right cosets $\sum_{\theta=1}^{h} H S_{t} H_{\theta} G_{k}$ in which, as we shall see in $\$ 4$, each of the $k_{t}$ cosets occurs $d_{t}=h / k_{t}$ times. Its matrix $d_{t} V_{t}$ is permutable with each of the matrices of $G_{H}$. Certain cosets, which we shall call associated cosets, are permuted among themselves when multiplied on the right by elements of $H$. Each of these produces the same matrix $V_{t}$. The totality $K_{t}$ of elements belonging to a complete set of $k_{t}$ associated cosets, each counted once, will be called a double coset, whereas the term weighted double coset will refer to the complex of $h^{2}$ elements $H S_{t} H$ in which each element of the double coset $K_{t}$ occurs $d_{t}=n / k_{t}$ times. The integer $d_{t}$ will be called the density. The number of distinct double cosets $K_{t}$ will be denoted by $r$, and the elements $S_{t}$, $t=1,2, \cdots, r$, one from each, will be said to generate the double

\footnotetext{
${ }^{1}$ Presented to the Society, September 12, 1940.

$2 \mathrm{~J}$. S. Frame, The degrees of the irreducible components of simply transitive permutation groups, Duke Mathematical Journal, vol. 3 (1937), pp. 8-17.

J.S. Frame, On the decomposition of transitive permutation groups generated by the symmetric group, Proceedings of the National Academy of Sciences, vol. 26 (1940), pp. 132-139.
} 
cosets. The double coset which contains the inverses of the elements of $K_{t}$ will be denoted by $K_{t^{\prime}}$.

In $\$ 2$ a number of elementary properties of double cosets are stated, mostly without proof. In $\$ 3$ a study is made of self-inverse double cosets, $K_{t}=K_{t^{\prime}}$, and their number is shown to equal $(1 / g) \sum \chi\left(R^{2}\right)$, where $\chi\left(R^{2}\right)$ is the trace of $R^{2}$ in the permutation group $G_{H}$, and where the sum is taken for all $R$ in $G$. Using the results of a paper of Frobenius $^{3}$ we then prove the first principal theorem, which generalizes a result obtained by the author for the symmetric group. ${ }^{4}$

THEOREM A. The number of self-inverse double cosets of a finite group $G$ with respect to a subgroup $H$ is equal to the sum of the multiplicities of those irreducible components of $G_{H}$ which have a symmetric bilinear invariant minus the sum of the multiplicities of those which have an alternating bilinear invariant.

The Hermitian invariants of $G_{H}$ associated with the matrices $V_{t}$ are studied in $\$ 4 .{ }^{5}$ Two bases consisting of $r$ independent invariants are found, the one obtained directly from the $r$ double cosets, and the other from the $r=\sum_{i=1}^{r}\left(\mu_{i}^{H}\right)^{2}$ Hermitian invariants ${ }^{6}$ which come into evidence when $G_{H}$ is completely reduced. The complex multiplication of the double cosets plays an important role in the discussion which culminates in the proof of Theorem B, which includes as a special case a theorem conjectured but only partially proved in a previous paper. ${ }^{7}$

Theorem B. Given a transitive permutation group $G_{H}$ of degree $n$ in which the subgroup $H$ leaving one symbol fixed permutes the $n$ symbols in $r$ transitive sets of $k_{t}$ symbols, $t=1,2, \cdots, r$. Let $K$ be the product $\prod_{t=1}^{r}\left(k_{t}\right)$ and let $N, N=\prod_{i=1}^{r} n_{i}^{\left(\mu_{i}^{H}\right)^{2}}$, denote the product of the degrees $n_{i}$ of the distinct irreducible components $\Gamma_{i}$ of $G_{H}$ each raised to a power equal to the square of its multiplicity in $G_{H}$. Then $n^{r-2} K / N=\bar{P}_{1} P_{1}$, where $P_{1}$ is an algebraic integer in the field of the characters of the components of $G_{H}$.

${ }^{3}$ G. Frobenius and I. Schur, Über die reellen Darstellungen der endlichen Gruppen, Sitzungsberichte der Preussischen Akademie der Wissenschaften, 1906 (I), pp. 186208, especially p. 197. The author is indebted to G. de B. Robinson for referring him to this article.

${ }^{4}$ J. S. Frame, Proceedings of the National Academy of Sciences, loc. cit.

${ }^{5}$ A study of these matrices was made by I. Schur, Zur Theorie der einfach transitiven Permutationsgruppen, Sitzungsberichte der Preussischen Akademie der Wissenschaften, 1933, pp. 598-623.

${ }^{6}$ This relation is well known in group theory. See, for example, W. Burnside, Theory of Groups, 1911, p. 275.

7 J. S. Frame, Duke Mathematical Journal, loc. cit. 
In $\$ 5$ a study of the bilinear invariants of the components of $G_{H}$ which are irreducible in the real domain leads to an alternate proof of Theorem A, based on methods similar to those of $\$ 4$.

2. Elementary properties of double cosets. The set of elements permutable with a coset $H S_{i}$ form a group $N_{i}$ called the normalizer of the coset, whose intersection with $H$ is a group $D_{i}$ of order $d_{i}$ leaving $H S_{i}$ fixed in the permutation group $G_{H}$. We let $D_{i} H_{i j}$ be the $k_{i}=h / d_{i}$ cosets of $H$ with respect to $D_{i}$ and note that for each $j$ the elements $H S_{i} D_{i} H_{i j}=H\left(S_{i} H_{i j}\right)$ form a different coset $H S_{j}$ associated with $H S_{i}$. Hence there are $k_{t}$ distinct cosets each occurring $d_{t}$ times in the weighted double coset $H S_{t} H$. If $H$ is invariant in $G$, then each $D_{t}=H$, each $k_{t}=1$, and each coset is a double coset. It is convenient to select the $S_{j}$ for associated cosets so that $S_{i} H_{i j}=S_{j}$ and then to define $H_{j i}=S_{j}^{-1} S_{i}=H_{i j}^{-1}$, when obtaining the cosets of $H$ with respect to its subgroup $D_{j}=H_{i j}^{-1} D_{i} H_{i j}$, which is permutable with $H S_{j}$. It is seen immediately that the subgroups $D_{i}, D_{j}, \cdots$ which are permutable with the right cosets $H S_{i}, H S_{j}, \cdots$ of a double coset $H S_{t} H$ are all of the same order $d_{t}$ and form a complete set of conjugate subgroups of $H$.

The following four properties of double cosets are simple enough to be given here without proof. ${ }^{8}$

THEOREM 2.1. Each element of $G$ lies in one and only one double coset of $G$ with respect to a given subgroup $H$.

Theorem 2.2. A double coset may be generated by any one of its elements.

TheOREM 2.3. The inverses $H_{j}^{-1} S_{t}^{-1} H_{i}^{-1}$ of the elements $H_{i} S_{t} H_{j}$ of the double coset $K_{t}$ form a double coset $K_{t^{\prime}}$ generated by $S_{t}^{-1}$, which has the same density as $K_{t}$.

If $K_{t}=K_{t^{\prime}}$, the double coset is called self-inverse.

THEOREM 2.4. A double coset which contains a self-inverse element is self-inverse. In particular the double coset $H=K_{1}$ is self-inverse.

The next three theorems show that the elements of a class of conjugates, of a left coset, and of the set of inverses of a right coset, are equally distributed among the right cosets of their double coset.

ThEOREM 2.5. Each coset of a double coset $K_{t}$ contains the same num-

${ }^{8}$ Several of the theorems in $\$ 2$ are implied in the discussion of cosets in the standard texts on group theory. Nowhere has the author found them collected as properties of double cosets as such. They are stated here for convenience of reference for the later proofs. 
ber $g_{t \lambda}$ of the $g_{\lambda}$ elements forming the class $C_{\lambda}$ of conjugate elements in $G .{ }^{9}$

Proof. Let $H_{\theta} S_{i}$ be a common element of $H S_{i}$ and $C_{\lambda}$, and let the subgroup of $D_{i}$ which is permutable with the element $H_{\theta} S_{i}$ be of index $g_{i \lambda}$ in $D_{i}$. Then there will be $g_{i \lambda}$ distinct elements each counted $d_{i} / g_{i \lambda}$ times among the elements $\delta_{\kappa}^{-1}\left(H_{\theta} S_{i}\right) \delta_{\kappa}, \delta_{\kappa}$ in $D_{i}$. Each of these distinct elements of $H S_{i}$ is transformed by $H_{i j}$ into a different element of $H S_{j}$, so the numbers $g_{i \lambda}, g_{j \lambda}, \cdots$ are the same for all the cosets of $K_{t}$ and may be denoted by $g_{t \lambda}$.

TheOREM 2.6. The $h$ elements of the left coset $\left(H_{\theta} S_{i}\right) H$ are equally distributed among the $k_{i}$ right cosets associated with $H S_{i}$, just $d_{i}$ elements lying in each right coset.

Proof. Each element of $H$ can be written uniquely in the form $\delta_{\kappa} H_{i j}$, where $\delta_{\kappa}$ is in $D_{i}$. For each $j$ we obtain $d_{i}$ elements $H_{\theta} S_{i} \delta_{\kappa} H_{i j}$ $=\left(H_{\theta} S_{i} \delta_{k} S_{i}^{-1}\right) S_{j}$ in $H S_{j}$.

If we note further that the inverses of the elements of a right coset $H S_{i}$ form a left coset $S_{i}^{-1} H$, then we obtain the following:

THEOREM 2.7. The inverses of the elements of a right coset are equally distributed among the $k_{i}$ cosets of the inverse double coset, with $d_{i}$ elements in each. Furthermore each coset of a self-inverse double coset $K_{t}$ contains just $d_{t}$ of its own inverses.

THEOREM 2.8. Each element of the normalizer $N_{1}$ of $H$ transforms a double coset $K_{t}$ into itself or into a double coset $N_{1}^{-1} K_{t} N_{1}$ having the same distribution of elements among the classes $C_{\lambda}$ as $K_{t}$ does.

These double cosets may be called conjugate double cosets.

Proof. Each element is transformed into a conjugate element in the double coset generated by $N_{1}^{-1} S_{t} N_{1}$.

THEOREM 2.9. (a) The number of times, $k_{t u v}$, that the identity element $E$ occurs among the $h^{3}$ elements $H S_{t} H S_{u} H S_{v}$ depends only on the double cosets to which $S_{t}, S_{u}$, and $S_{v}$ belong.

(b) The constant $k_{t u v}$ is unchanged by an even permutation of the double cosets $K_{t}, K_{u}, K_{v}$, or by an odd permutation coupled with a change to inverse double cosets.

(c) The number of the $h$ elements $S_{u} H S_{v}$ which lie in the double coset $K_{t^{\prime}}$ is $k_{t u v} / d_{t}$, and $\sum_{t=1}^{r} k_{t u v} / d_{t}=h$.

(d) For $t=1$, we have $k_{1 u v}=k_{u v 1}=h d_{u} \delta_{u v^{\prime}}$.

${ }^{9}$ D. E. Littlewood, Theory of Group Ckaracters, 1940, p. 149. Littlewood shows, if we change his notation to ours, that the characters in $G_{H}$ of the elements of $C_{\lambda}$ are $n g_{1 \lambda} / g_{\lambda}$. 
(e) The product of the double cosets $K_{t} K_{u}$ is given by the equation

$$
K_{t} K_{u}=\left(h /\left(d_{t} d_{u}\right)\right) \sum_{v} k_{t u v^{\prime}} K_{v}
$$

To prove the various parts of Theorem 2.9, let us assume that $H_{a}, H_{b}$, and $H_{c}$ are elements of $H$ which satisfy the equation $H_{a} S_{t} H_{b} S_{u} H_{c} S_{v}=E$. The equation is still valid if we permute the six elements cyclicly, or if we interpose between two adjacent ones the product of an element of $H$ and its inverse, thus replacing an element by another element in its double coset, or if we replace all six elements by their inverses in the opposite order. The remainder of the proof depends on eliminating the weighting factors from the weighted double cosets $H S_{t} H$, etc., but may be left to the reader.

3. The number of self-inverse double cosets. We shall now apply the theorems of $\$ 2$ to obtain expressions for the number of self-inverse double cosets of a group.

THEOREM 3.1. The number $N_{H}$ of self-inverse double cosets $K_{t}$ of $G$ with respect to $H$ is $1 / \mathrm{g}$ times the number of solutions of the equation

$$
S_{j} R^{2}=H_{k} S_{j}, \quad j=1,2, \cdots, n ; H_{k} \text { in } H, R \text { in } G .
$$

We rewrite the equation in the form $H_{k}^{-1}\left(S_{j} R S_{j}^{-1}\right)=\left(S_{j} R S_{j}^{-1}\right)^{-1}$, which states that the inverse of the element $S_{j} R S_{j}^{-1}=T$ belongs to its own right coset. For each of the $k_{t}$ cosets of $K_{t}$ there are $d_{t}$ available values of $T$, by Theorem 2.7. For each of these $h=d_{t} k_{t}$ values of $T$ we may choose $n$ generators $S_{j}$ to determine an element $R=S_{j}^{-1} T S_{j}$ conjugate to $T$. Thus for each self-inverse double coset we obtain $g=n h$ solutions of the given equation, and the theorem is proved.

Now the permutation matrix corresponding to $R^{2}$ in $G_{H}$ has a unit in the principal diagonal for every right coset which remains fixed by $R^{2}$, that is, for every solution of the equation $S_{j} R^{2}=H_{k} S_{j}, H_{k}$ in $H$, $j=1,2, \cdots, n$. Its trace is the number of these solutions for fixed $R$. When we sum these for all $R$ in $G$ and apply Theorem 3.1, we obtain the following theorem.

THEOREM 3.2. The number $N_{H}$ of self-inverse double cosets of $G$ with respect to $H$ is given by the formula

$$
N_{H}=(1 / g) \sum_{R} \chi\left(R^{2}\right),
$$

where $\chi(S)$ is the trace of the matrix $S$ in the permutation group $G_{H}$.

This theorem may be applied to the results of a paper by Frobenius 
and Schur ${ }^{10}$ to obtain the fundamental Theorem A of $\$ 1$. It is known in the theory of group characters that for an irreducible representation $\Gamma_{i}$ with character $\chi^{i}(R)$ the quantity

$$
\sum_{R}\left\{\left[\chi^{i}(R)\right]^{2}+\chi^{i}\left(R^{2}\right)\right\} /(2 g)
$$

is 1 or 0 according as $\Gamma_{i}$ has or has not a symmetric bilinear invariant, and that

$$
\sum_{R}\left\{\left[\chi^{i}(R)\right]^{2}-\chi^{i}\left(R^{2}\right)\right\} /(2 g)
$$

is 1 or 0 according as $\Gamma_{i}$ has or has not an alternating bilinear invariant. Following Frobenius ${ }^{10}$ we set $c_{i}=1,-1$, or 0 according as $\Gamma_{i}$ has a symmetric, an alternating, or no bilinear invariant. We shall call these respectively "symmetric," "quaternion," and "rotary" representations. Then

$$
(1 / g) \sum_{R} \chi^{i}\left(R^{2}\right)=c_{i}
$$

Now let $G_{H}$ be reduced into irreducible components $\Gamma_{i}$ with multiplicities $\mu_{i}^{H}$. Then

$$
G_{H}=\sum_{i} \stackrel{H}{\mu_{i}} \Gamma_{i}, \quad \chi(R)=\sum_{i} \underset{\mu_{i}}{H} \chi^{i}(R) .
$$

Hence, by (3.3), (3.7), and (3.6), we have

$$
N_{H}=(1 / g) \sum_{R} \chi\left(R^{2}\right)=(1 / g) \sum_{R} \sum_{i} \stackrel{H}{\mu_{i}} \chi^{i}\left(R^{2}\right)=\sum_{i} c_{i} \mu_{i}^{H} .
$$

This formula is equivalent to Theorem $\mathrm{A}$ of $\$ 1$.

4. A theorem derived from a unitary reduction of a group and its double cosets. When a group $G$ is represented in two ways as a regular permutation group of degree $g$, using cosets with respect to the identity subgroup $E$, the one $G_{E}$ obtained by the right multiplication of cosets by the elements of $G$, and the other $G_{E}^{\prime}$ by left multiplication, the matrices of $G_{E}{ }^{\prime}$ form a basis for all matrices permutable with those of $G_{E}$, and vice versa. In that case each of the elements of $G$ forms a double coset. But when cosets are taken with respect to a subgroup $H \neq E$, a basis for the matrices permutable with those of the right multiplication permutation group $G_{H}$ is found in the double cosets of the left multiplication group. The matrices $V_{t}$ described in $\$ 1$ are

$10 \mathrm{G}$. Frobenius and I. Schur, loc. cit. 
those of a complete set of invariant Hermitian forms of $G_{H}$ on which all others are linearly dependent. For let $x_{i}, x_{i}=x\left(H S_{i}\right), i=1,2, \cdots, n$, be the variables permuted by $G_{H}$. Then the matrix $R$ of $G_{H}$ transforms the product $\bar{x}(H) x\left(H S_{t}\right)$ into $\bar{x}(H R) x\left(H S_{t} R\right)$, and if we write $R=H_{j} G_{k}$ and sum over all $R$ in $G$, we obtain an Hermitian form invariant under the right multiplications of $G_{H}$. Since the subgroup $D_{t}$ of $H$ leaves $H S_{t}$ fixed, each term of this form will contain the factor $d_{t}$. Dividing by $d_{t}$ and writing $K_{t}=H S_{t} H / d_{t}$, we obtain the simpler invariant Hermitian form $\sum_{k} \bar{x}\left(H G_{k}\right) x\left(K_{t} G_{k}\right)$, whose matrix we have denoted by $V_{t}$. The matrix $V_{1}$ is the unit matrix. Each row and column of $V_{t}$ contains $k_{t} 1$ 's and the rest 0 's, and the sum of all the matrices $V_{t}$ is a matrix consisting entirely of $1^{1}$ 's. ${ }^{11}$ To the inverse double coset $K_{t^{\prime}}$ corresponds the transposed matrix $V_{t}^{\prime}$, which will be denoted by $V_{t^{\prime}}$. Self-inverse double cosets have symmetric matrices $V_{t}$.

Now let $U$ be a unitary matrix which completely reduces the group $G_{H}$ into its irreducible components $\Gamma_{i}$ of degree $n_{i}$ and multiplicity $\mu_{i}^{H}$, so that all equivalent components of $U^{-1} G_{H} U$ are actually identical and so that the invariant Hermitian form for each component is a diagonal form with unit matrix $E_{i}$ of degree $n_{i}$. Then for the set of $\mu_{i}^{B}$ equal components $\Gamma_{i}$ we have $\left(\mu_{i}^{H}\right)^{2}$ linearly independent Hermitian forms whose matrices $\sum_{\alpha \beta} E_{i}^{\alpha \beta} \rho_{i}^{\alpha \beta}$ are obtained as the direct product of $E_{i}$ with an arbitrary matrix $\left(\rho_{i}^{\alpha \beta}\right)$ of degree $\mu_{i}^{H}$. The $r$ matrices $M_{t}=U^{-1} V_{t} U$, obtained by transforming the Hermitian invariants of $G_{H}$, must be expressible as linear combinations of the $r$ matrices $E_{i}^{\alpha \beta}$. It is convenient to arrange the symbols $E_{i}^{\alpha \beta}$ in some arbitrary order starting with $E_{1}^{11}=E_{1}$, and to assign to each a single subscript $\gamma$. We write

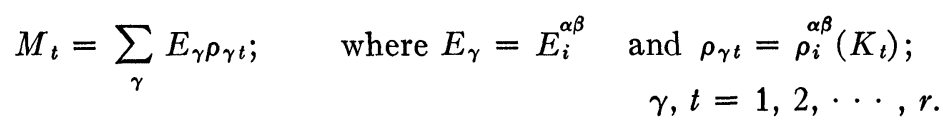

Since the matrices $M_{t}$ and $E_{\gamma}$ each form a basis for the invariant Hermitian forms of $U^{-1} G_{H} U$, the matrix $\left(\rho_{\gamma t}\right)$ is nonsingular.

The $M_{t}$ combine according to a rule similar to the rule of combination for the inverse double cosets $K_{t^{\prime}}$, as given in 2.10 , namely,

$$
M_{t} M_{u}=\sum_{v} c_{t u v} M_{v}, \quad c_{t u v}=k_{t^{\prime} u^{\prime} v} /\left(d_{t} d_{u}\right) .
$$

Since this is also the rule of combination for the matrices $\rho_{i}^{\alpha \beta}\left(K_{t}\right)$ corresponding to a given $\Gamma_{i}$, these matrices give that representation for the left multiplication of double cosets which is associated with $\Gamma_{i}$.

${ }^{11}$ J. S. Frame, Duke Mathematical Journal, loc. cit. 
Now when $G_{E}$ and $G_{E}^{\prime}$ are simultaneously reduced, the component of $G_{E}^{\prime}$ on the same variables as the $n_{i}$ components $\Gamma_{i}$ of $G_{E}$ consists of the direct product of $E_{i}$ with a representation equivalent to $\Gamma_{i}^{\prime}$. When the matrices of this representation are summed over all elements in each double coset, the resulting set of $r$ matrices when reduced will contain the component of degree $\mu_{i}^{H} \leqq n_{i}$, expressible in terms of the variables of $G_{H}$, which is equivalent to the matrices $\rho_{i}^{\alpha \beta}\left(K_{t}\right)$. Hence the $\rho_{i}^{\alpha \beta}\left(K_{t}\right)$ are expressible in terms of the matrices of $\Gamma_{i}^{\prime}$.

The rule (4.2) is also the rule of combination for the matrices $V_{t},{ }^{12}$ so the coefficients $c_{t u v}$ must be integers, and the character of the product $M_{t} M_{u}$ is given by the formula

$$
\chi\left(M_{t^{\prime}} M_{u}\right)=n c_{t^{\prime} u 1}=n k_{t} \delta_{t u},
$$

in view of Theorem 2.9 (d). Since $U$ is unitary, the matrix $M_{t}$, is the transposed conjugate $\bar{M}_{t}^{\prime}$ of $M_{t}$. We shall denote by $E_{\gamma^{\prime}}$ the transposed conjugate of $E_{\gamma}$, and by $n_{\gamma}$ the degree of the corresponding irreducible representation.

Next consider the matrices $\left(M_{t^{\prime} u}\right)$ and $\left(E_{\gamma^{\prime} \delta}\right)$ of degree $r$ whose elements are

$$
\begin{aligned}
M_{t^{\prime} u} & =\chi\left(M_{t^{\prime}} M_{u}\right)=\chi\left(V_{t^{\prime}} V_{u}\right)=n k_{t} \delta_{t u}, \\
E_{\gamma^{\prime} \delta} & =\chi\left(E_{\gamma^{\prime}} E_{\delta}\right)=n_{\gamma} \delta_{\gamma \delta} .
\end{aligned}
$$

We obtain a relation between $\left(M_{t^{\prime} u}\right)$ and $\left(E_{\gamma^{\prime} \delta}\right)$ as follows:

$$
M_{t^{\prime} u}=\chi\left(\sum_{\gamma} \bar{\rho}_{t \gamma} E_{\gamma^{\prime}} \sum_{\delta} E_{\delta} \rho_{\delta u}\right)=\sum_{\gamma, \delta} \bar{\rho}_{t \gamma} E_{\gamma^{\prime} \delta} \rho_{\delta u} .
$$

Denoting the determinant of $\left(\rho_{\delta u}\right)$ by $P$, we have, by $(4.4),(4.5),(4.6)$,

$$
\prod_{t=1}^{r}\left(n k_{t}\right)=\left(\prod_{\gamma=1}^{r} n_{\gamma}\right) \bar{P} P=\left(\prod_{i=1}^{r^{\prime}} n_{i}^{\left(\mu_{i}^{H}\right)^{2}}\right) \bar{P} P .
$$

Since $\sum_{t} \rho_{\gamma t}=n \delta_{\gamma 1}$, the determinant $P$ may be written in the form $n P_{1}$, where $P_{1}$ is the minor of $\rho_{11}$ in $P$. Factoring $n^{2}$ from both sides of (4.7) and using the notation of Theorem $B$, we have

$$
n^{r-2} K=N \bar{P}_{1} P_{1}, \quad \text { where } \quad K=\prod_{t=1}^{r} k_{t}, \quad N=\prod_{i=1}^{r^{\prime}} n_{i}^{\left(\mu_{i}^{H}\right)^{2}} .
$$

Since for each $i$ the matrices $\rho_{i}^{\alpha}\left(K_{t}\right)$ give a representation of the ring of matrices $V_{t}$ in which the coefficients of combination are integers, the matrix $P_{1}$ is an algebraic integer. It belongs to the field of char-

${ }^{12}$ J. S. Frame, Duke Mathematical Journal, loc. cit. 
acters of the $\Gamma_{i}$. This completes the proof of Theorem B. It will be noted that if the representations $\Gamma_{i}$ have rational characters, which will certainly be true if their degrees are all distinct, then the quotient $n^{r-2} \mathrm{~K} / N$ will be a perfect square.

5. A theorem derived from a real orthogonal reduction of a group and its double cosets. Let us now consider the reduction of $G_{H}$ by a real orthogonal matrix $O$ into a form which is irreducible in the real domain. The component representations are of three types: (1) Those "symmetric" representations $\Gamma_{i}^{(+)}$which are absolutely irreducible in the complex domain and have a symmetric bilinear invariant. (2) Those "quaternion" representations $2 \Gamma_{i}^{(-)}$which consist of two equivalent complex components of even degree each with real characters and each with an alternating but not a symmetric bilinear invariant. The pair together have a third alternating bilinear invariant and a symmetric bilinear invariant. The matrices of the four invariants, suitably normalized, combine like the quaternion units. (3) Those "rotary" representations $\Gamma_{i}^{(0)}+\Gamma_{i}^{(0)}$ which have two non-equivalent conjugate complex absolutely irreducible components, each having complex characters, but having no bilinear invariant. Taken together they have an alternating and a symmetric bilinear invariant which, when suitably normalized, combine like the real and imaginary units.

The matrices $O^{-1} V_{t} O$ form a basis for the invariant bilinear forms, but in place of the other basis matrices $E_{\gamma}$ used in $\$ 4$, we now use matrices $E_{\gamma}$ which for the symmetric representations of type 1 are like the old $E_{\gamma}$, for the quaternion representations of type 2 come in sets of four which multiply like the quaternion units, and for the rotary representations of type 3 come in pairs which multiply like the real and imaginary units. Those of type 2 are as follows:

$$
\begin{aligned}
& \left(\begin{array}{cccc}
e_{i} & 0 & 0 & 0 \\
0 & e_{i} & 0 & 0 \\
0 & 0 & e_{i} & 0 \\
0 & 0 & 0 & e_{i}
\end{array}\right), \\
& \left(\begin{array}{rrrr}
0 & 0 & e_{i} & 0 \\
0 & 0 & 0 & e_{i} \\
-e_{i} & 0 & 0 & 0 \\
0 & -e_{i} & 0 & 0
\end{array}\right) \\
& \left(\begin{array}{rrrc}
0 & -e_{i} & 0 & 0 \\
e_{i} & 0 & 0 & 0 \\
0 & 0 & 0 & e_{i} \\
0 & 0 & -e_{i} & 0
\end{array}\right), \\
& \left(\begin{array}{rccc}
0 & 0 & 0 & e_{i} \\
0 & 0 & -e_{i} & 0 \\
0 & e_{i} & 0 & 0 \\
-e_{i} & 0 & 0 & 0
\end{array}\right),
\end{aligned}
$$

where $e_{i}$ denotes the unit matrix of degree $n_{i} / 2$. 
We next normalize each of the matrices $V_{t}$ and $E_{\gamma}$ by dividing by the square root of the sum of the squares of its coefficients. From the new normalized matrices $V_{t}^{*}$ and $E_{\gamma}{ }^{*}$, thought of as of degree $n$, we form a new set of matrices of degree $r$ defined by

$$
\begin{array}{ll}
V_{t u}^{*}=\chi\left(V_{t}^{*} V_{u}^{*}\right), & V_{t^{\prime} u}^{*}=\chi\left(V_{t}^{* \prime} V_{u}^{*}\right), \\
E_{\gamma \delta}^{*}=\chi\left(E_{\gamma}^{*} E_{\delta}^{*}\right), & E_{\gamma^{\prime} \delta}^{*}=\chi\left(E_{\gamma}^{* \prime} E_{\delta}^{*}\right) .
\end{array}
$$

Then $\left(V_{t^{\prime} u}^{*}\right)$ and $\left(E_{\gamma^{\prime} \delta}^{*}\right)$ are each the unit matrix, whereas $\left(V_{t u}^{*}\right)=\left(\delta_{t^{\prime} u}\right)$ is a matrix whose trace is the number $N_{H}$ of self-inverse double cosets, and $\left(E_{\gamma \delta}^{*}\right)$ is a matrix whose trace is $\sum_{i=1}^{r} c_{i} \mu_{i}^{H}$, since each component $\Gamma_{i}^{(+)}$of type 1 contributes +1 , each real component $2 \Gamma_{i}^{(-)}$of type 2 containing a pair of equivalent irreducible representations contributes $+1-1-1-1=-2$, and each real component of type 3 contributes $+1-1=0$. Now let

$$
O^{-1} V_{t}^{*} O=\sum_{\gamma} E_{\gamma}^{*} q_{\gamma t}
$$

define a matrix $Q$ which changes the basis of the bilinear forms of $O^{-1} G_{H} O$. Then

$$
\begin{aligned}
V_{t u}^{*} & =\chi\left(V_{t}^{*} V_{u}^{*}\right)=\chi\left(O^{-1} V_{t}^{*} O \cdot O^{-1} V_{u}^{*} O\right)=\chi\left(\sum_{\gamma} E_{\gamma}^{*} q_{\gamma t} \cdot \sum_{\delta} E_{\delta}^{*} q_{\delta u}\right) \\
& =\sum_{\gamma, \delta} q_{\gamma t} E_{\gamma \delta}^{*} q_{\delta u} .
\end{aligned}
$$

Similarly, since $\left(V_{t^{\prime} u}^{*}\right)$ and $\left(E_{\gamma, \delta}^{*}\right)$ are unit matrices, we have

$$
V_{t^{\prime} u}^{*}=\sum_{\gamma, \delta} q_{\gamma^{\prime} t^{\prime}} E_{\gamma^{\prime} \delta} q_{\delta u}=\sum q_{\delta t^{\prime}} q_{\delta u}=\delta_{t^{\prime} u}
$$

Hence $Q$ is an orthogonal matrix, and (5.4) may be written in the form

$$
\left(V_{t u}^{*}\right)=Q^{-1}\left(E_{\gamma \delta}^{*}\right) Q .
$$

By equating the traces of $\left(V_{t u}^{*}\right)$ and $\left(E_{\gamma \delta}^{*}\right)$ given above, an alternate proof of Theorem A is obtained.

Brown UNIVERSITY 